\title{
Analysis of NMR Spectral Analysis and Molecular Electronic Potential Maps on L-Arginine Phosphate Crystals for Non-Linear Optical Applications
}

\author{
Chandran Tamil Malar 1(D), Parasuraman Kumaresan 1(D), Subramanian Nithiyanatntham 2,*(i), \\ Kuppusamy Sambathkumar ${ }^{3}$ (D) \\ 1 P.G \& Research Department of Physics, Thiru.A.Govindasamy Government Arts College, Tindivanam-604 307, Tamil \\ Nadu, India, tamilmalarchandran2021@gmail.com (C.T.M.); logeshkumaresan@yahoo.com (P.K.); \\ 2 P.G \& Research Department of Physics, Thiru.Vi.Ka. Government Arts College, Thiruvarur, Tamil Nadu, India, \\ s_nithu59@rediffmail.com (S.N.); \\ 3 P.G \& Research Department of Physics, Arignar Anna Government Arts College, Villupuram - 605 603, Tamil Nadu, \\ India, sa975kumar@gmail.com (K.S.); \\ * Correspondence: s_nithu59@rediffmail.com (S.N.);
}

Received: 20-10-2021; Revised: 14.11.2021; Accepted: 15-11-2021; Published: 11.12.2021

\begin{abstract}
L-Arginine Phosphate (LAP) is a possible material for applications involving nonlinear optical properties. Slow evaporation was used to generate single LAP crystals with a very high degree of transparency from an aqueous solution. The solubility of the pure and doped LAP crystals was measured at different temperatures in the double-distilled water. Natural bond orbital (NBO) research investigated the molecule's stability and charge delocalization. The HOMO-LUMO energies describe the charge transfer between molecules. The electrostatic potential of molecules has been investigated. The correlation found between crystalline perfection and SHG potency was mentioned.
\end{abstract}

Keywords: LAP crystals; HOMO-LUMO; NBO analysis; perturbation theory energy analysis; potential energy scan studies.

(C) 2021 by the authors. This article is an open-access article distributed under the terms and conditions of the Creative Commons Attribution (CC BY) license (https://creativecommons.org/licenses/by/4.0/).

\section{Introduction}

Several researchers are currently interested in nonlinear optical (NLO) materials because of their potential uses in future optoelectronic and photonic technologies [1-3]. Some technological challenges currently exist, such as the creation of blue light from a near-infrared (NIR) laser via Second Harmonic Generation (SHG) [4,5]. A nonlinear material, usually a crystal, is required for this type of nonlinear optical process. Investigation of improved nonlinear optical materials is required to overcome this serious challenge. The LAP crystal is important in the field of second harmonic generating devices and laser tuned experiments [6,7]. When a centrosymmetric LAP molecule reacts with inorganic material, it forms noncentrosymmetric (NLO) complexes, which contain organic and inorganic properties and are referred to as semi-organic material [8].

The highest occupied molecular orbital (HOMO) and the lowest unoccupied molecular orbital (LUMO) are critical factors in quantum chemistry. The computation is done by looking at all conceivable interactions between 'full' (donor) Lewis-type NBOs and 'empty' (acceptor) non-Lewis NBOs and using 2nd order perturbation theory to estimate their energetic relevance. As a result, LAP is a promising choice for nonlinear optical applications, as it has a higher 
SHG efficiency than KDP. The LAP crystal was created using a variety of procedures that necessitated the use of sophisticated tools.

\section{Materials and Methods}

Using the B3LYP/6 - 311+G(d,p) basis set and the finite field technique, the first hyperpolarizability $(0)$ of the molecular system and the corresponding characteristics $(0,0)$ of L-Arginine Phosphate (LAP) are computed. The energy of a system is a function of first hyperpolarizability, third-rank tensor that can be expressed by a 333 matrix in the presence of an applied electric field. The Kleinman symmetry $[9,10]$ allows the 27 components of the 3D matrix to be reduced to 10 components. When the energy in the external electric field is weak and homogeneous, the components are defined as the coefficients in the Taylor series expansion.

$$
\mathrm{E}=\mathrm{E}_{0}-\mu_{\alpha} \mathrm{F}_{\alpha}-1 / 2 \alpha_{\alpha \beta} \mathrm{F}_{\alpha} \mathrm{F}_{\beta}-1 / 6 \beta_{\alpha} \beta_{\gamma} \mathrm{F}_{\alpha} \mathrm{F}_{\beta} \mathrm{F}_{\gamma}+\ldots \ldots
$$

where, $\mathrm{E}_{0}$ is the energy of unperturbed molecules, $\mathrm{F}$ is the field at the origin, and,, and are the dipole moment, polarizability, and initial hyperpolarizabilities components, respectively. Using the $\mathrm{x}, \mathrm{y}$, and $\mathrm{z}$ components, calculate the total static dipole moment, mean polarizability 0 , and mean initial hyperpolarizability 0 [11].

Because the output of GAUSSIAN 09W is reported in atomic units, the estimated values are translated to e.s.u. units $\left(1\right.$ a.u. $=8.3693 \times 10^{-33}$ (e.s.u.). Table 1 shows the calculated values of hyperpolarizability and polarizability of LAP.

\section{Results and Discussion}

Hyperpolarizabilities are used to characterize organic compounds with conjugated electrons, and vibrational spectroscopy is used to investigate them [12]. However, intramolecular charge transfer from the donor to accepter acceptor group via a single-double bond conjugated channel can cause significant changes in molecular dipole moment and polarizability. The border orbitals are so named because they can be used to determine how a molecule interacts within a species. HOMO, which is assumed to be the outermost orbital containing electrons, tends to act as an electron donor for these electrons. On the other hand, the innermost orbital, LUMO, can be thought of as having free locations to accept electrons $[13,14]$.

With molecular orbital theory, a state transition of the * type is observed due to the interaction between a structure's HOMO and LUMO orbitals. As a result, while the HOMO's energy is proportional to its ionization potential, the LUMO's energy is proportional to its electron affinity. The energy gap is the difference in energy between the HOMO and LUMO orbitals, and it is an important structural stability factor (Figure 1). The replacement groups like $\mathrm{N}$ and oxygen have no effect on HOMO, which is located on the middle ring. The substituted electronegative oxygen atom contributes to LUMO, which is quite concentrated on the centering.

The energy gap between HOMO and LUMO is -0.1530 a.u., indicating that charge transfer from the ring to the oxygen atom is possible (Table1and Table 2). Global chemical reactivity descriptors of compounds such as hardness, chemical potential, softness, electronegativity, electrophilicity index, and local reactivity have been characterized using density functional descriptors. The notion of electronegativity was established by Linus 
Pauling, who defined it as the ability of an atom in a compound to attract electrons. The following are the definitions for hardness $(\eta)$, chemical potential $(\mu)$, electrophilicity index $(S)$, electronegativity $(\omega)$, and softness $(\chi)$.

$$
\begin{aligned}
\eta & =(\mathrm{I}-\mathrm{A}) / 2 \\
\mu & =-(\mathrm{I}+\mathrm{A}) / 2 \\
\mathrm{~S} & =1 / \eta \\
\omega & =\mu^{2} / 2 \eta \\
\chi & =-\mu=-(\partial \mathrm{E} / \partial \mathrm{N})_{\mathrm{V}(\mathrm{r})}(6)
\end{aligned}
$$

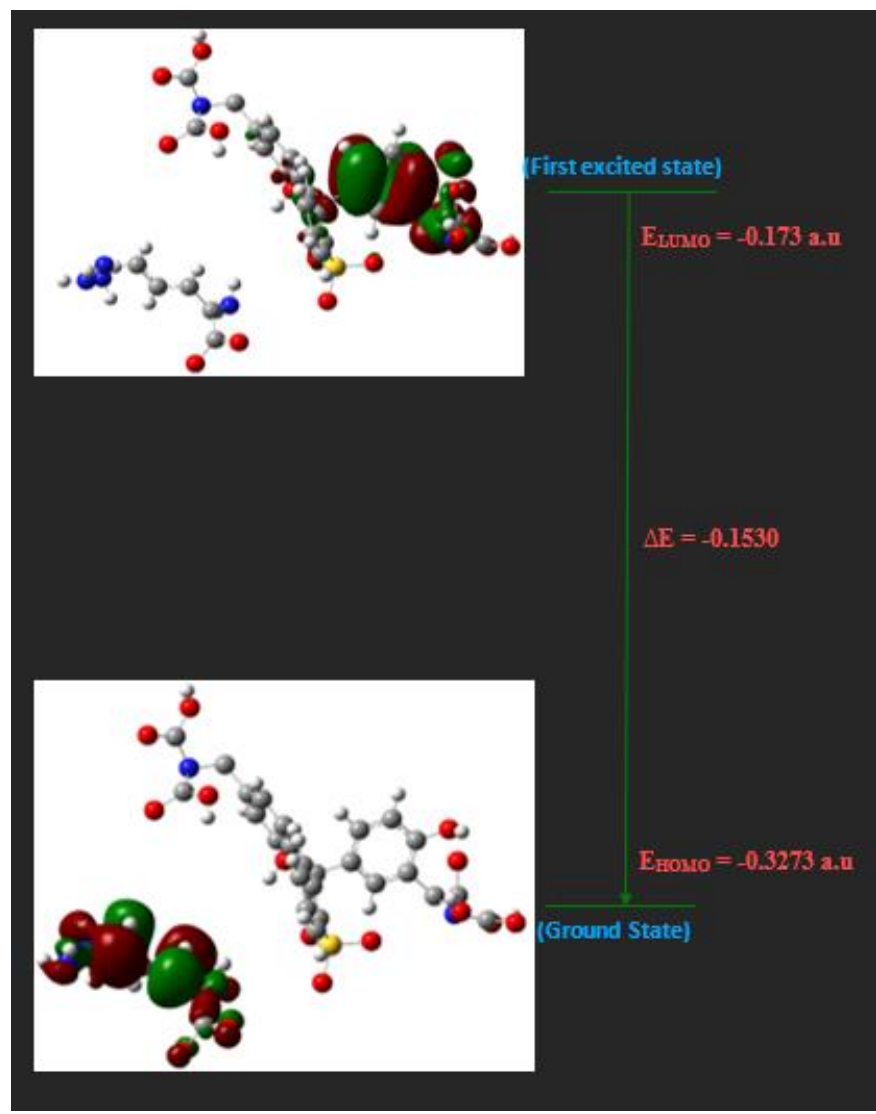

Figure. 1. HOMO-LUMO plot of LAP.

Table 1. Nonlinear optical properties of LAP calculated at B3LYP/6 - 311+G(d,p) method and basis set.

\begin{tabular}{l|c}
\multicolumn{1}{c|}{ NLO behavior } & B3LYP/6-311+G(d,p) \\
\hline Dipole moment $(\mu)$ & 3.1727 \\
\hline Mean polarizability $(\alpha)$ & $1.3793 \times 10^{-30}$ \\
\hline Anisotropy of the polarizability $\left(\Delta_{\alpha}\right)$ & $3.2723 \times 10^{-30}$ \\
\hline First hyperpolarizability $(\beta)$ & $3.0450 \times 10^{-30}$ \\
\hline Vector - first hyperpolarizability $\left(\beta_{\text {vec }}\right)$ & $1.8270 \times 10^{-30}$
\end{tabular}

Table 2. HOMO - LUMO energy gap and related molecular properties ofLAP.

\begin{tabular}{l|c}
\multicolumn{1}{c}{ Molecular Properties } & B3LYP/6 - 311+G(d,p) \\
\hline HUMO & -0.3273 \\
\hline LUMO & -0.173 \\
\hline Energy gap & -0.1530 \\
\hline Ionization Potential $(\mathrm{I})$ & 0.2565 \\
\hline Electron affinity $(\mathrm{A})$ & 0.0922 \\
\hline Global Hardness $(\eta)$ & 0.08215 \\
\hline Chemical potential $(\mu)$ & -0.17435 \\
\hline Global Electrophilicity $(\omega)$ & -0.2063
\end{tabular}




\section{Natural Bond Orbital Analysis}

Natural bond orbital (NBO) approaches are a set of algorithms that use computations to extract fundamental bonding principles from Density Functional Theory (DFT). Table 3 shows the atomic charges, core, valences, and total charge of LAP determined by NBO analysis using the $\mathrm{B} 3 \mathrm{LYP} / 6-31+\mathrm{G}(\mathrm{d}, \mathrm{p})$ technique. The negative charges of the ring carbon atoms $\mathrm{C}_{3}, \mathrm{C}_{5}$, $\mathrm{C}_{2}$, and $\mathrm{C}_{6}$ for LAP are the same.

However, due to the resonance of LAP, $\mathrm{N}_{17}, \mathrm{~N}_{21}, \mathrm{~N}_{5}$, and $\mathrm{N}_{31}$ have higher negative charges. For LAP, the costs for $\mathrm{H}_{7}, \mathrm{H}_{8}$, and $\mathrm{H}_{9}$ are the same. Table 4 lists the natural atomic orbitals, their occupancies, and LAP's corresponding energy. Natural atomic orbitals reflect the chemical give and take of electronic interactions in a given molecular environment, with differences in shape (e.g., angular deformations due to steric pressures of adjacent atoms) and size (e.g., altered diffuseness due to increased 250 anionic or cationic characters) that distinguish them appreciably from free atom forms. The orbital energies of NAOs I (A) are determined using the Kohn-Sham operator $(\mathrm{F})$ as follows:

$$
\varepsilon_{\mathrm{i}}^{(\mathrm{A})}=\left\langle\theta_{\mathrm{i}}^{(\mathrm{A})}|\mathrm{F}| \theta_{\mathrm{i}}^{(\mathrm{A})^{*}}\right\rangle
$$

The NAOs deal with interatomic and intraatomic contributions to molecular characteristics. Table 5 depicts bonding concepts such as orbital bond type, occupancies, natural atomic hybrids from which the NBO is composed, giving the percentage of the NBO on each hybrid, the atom label, and a hybrid label showing the hybrid orbital ( $\mathrm{spx}$ ) composition (the amount of s-character, $\mathrm{p}$-character, etc.) of the LAP molecule determined by B3LYP/6$31+\mathrm{G}(\mathrm{d}, \mathrm{p})$ method with respectable accuracy. In this hypothetical Lewis structure, the hybridization of the atoms and the weight of each atom in each localized electron-pair bond are calculated and reported in Table 5. The structure of LAP is well explained by typical Lewis electron-pair orbitals. Hence no antibonding orbitals are included [15].

\subsection{Donor acceptor interactions: perturbation theory energy analysis.}

The localized orbitals in LAP's Lewis structure can have many interactions. A donor can be a filled bonding or lone pair orbital, and an acceptor can be an empty or filled bonding, antibonding, or lone pair orbital. These exchanges have the potential to enhance or weaken bonds. A lone pair donor-antibonding acceptors orbital interaction, for example, could decrease the connection associated with the antibonding orbital 251. An interaction with a bonding pair as the acceptor, on the other hand, may enhance the bond. Donor-acceptor interactions are another manifestation of strong electron delocalization in the Lewis structure. Table 5 lists the stabilizing energy of various interactions for LAP, respectively.

Atomic charge, Lewis structure, bond type, hybridization, bond order, charge transfer, and resonance weights are all demonstrated using the NBO technique. Within the molecule, natural bond orbital (NBO) analysis is a useful technique for evaluating electron density delocalization from occupied Lewis-type (donor) NBOs to appropriately unoccupied nonLewis type (acceptor) NBOs. The energy differential between interacting orbitals is proportional to the stability of orbital interaction. As a result, the interaction with the highest level of stability occurs between effective donors and effective acceptors. The NBO technique, which is stated in terms of second-order perturbation interaction energy $\mathrm{E}^{(2)}$ [16-18], can be used to quantify this bonding-antibonding interaction quantitatively. The estimate of the offdiagonal NBO Fock matrix element is represented by this energy (Figure 6). The second-order 
perturbation technique [19-21] is used to quantify the stabilization energy $\mathrm{E}^{(2)}$ associated with I (donor) $\mathrm{j}$ (acceptor) delocalization.

$$
\mathrm{E}^{(2)}=\Delta \mathrm{E}_{\mathrm{ij}}=\mathrm{q}_{\mathrm{i}} \frac{\mathrm{F}(\mathrm{i}, \mathrm{j})^{2}}{\varepsilon_{\mathrm{j}}-\varepsilon_{\mathrm{i}}}
$$

The donor orbital occupancy is qi, the diagonal elements (orbital energies) are I and $\mathrm{j}$, and the off-diagonal Fock matrix element is $\mathrm{F}(\mathrm{i}, \mathrm{j})$. In the bond pair donor orbital, the $\mathrm{CC}^{*} \mathrm{NC}$ interaction between the $\mathrm{C}_{2}-\mathrm{C}_{3}$ bond pair and the antiperiplanar $\mathrm{C}_{1}-\mathrm{C}_{6}$ antibonding orbital stabilizes LAP at $6.86 \mathrm{~kJ} \mathrm{~mol}^{-1}$, while the $\mathrm{CC} * \mathrm{CH}$ interaction between the $\mathrm{C}_{1}-\mathrm{C}_{2}$ bond pair and the antiperiplanar $\mathrm{H}_{10}-\mathrm{C}_{16}$ antibonding orbital stabilizes LAP at $14.04 \mathrm{~kJ} \mathrm{~mol}^{-1}$. The bond pair donor orbital, $\mathrm{CH}^{*} \mathrm{NC}$ interaction between the $\mathrm{C}_{2}-\mathrm{H}_{8}$ bond pair and the antiperiplanar $\mathrm{C}_{1}-\mathrm{C}_{6}$ antibonding orbital, stabilizes LAP at $2.21 \mathrm{~kJ}$ mol-1, while the bond pair donor orbital, $\mathrm{CC} * \mathrm{NN}$ interaction between the $\mathrm{C} 2-\mathrm{C} 3$ bond pair and the antiperiplanar $\mathrm{N}_{17}-\mathrm{N}_{31}$ antibonding orbital stabilizes LAP at $2.41 \mathrm{~kJ} \mathrm{~mol}^{-1}$.

Table 3. Calculated atomic charges of LAP by natural bond orbital (NBO) analysis by B3LYP methods and

\begin{tabular}{|c|c|c|c|c|}
\hline Atom No & Charge & $\begin{array}{l}\text { basis set } \\
\text { Core }\end{array}$ & Valence & Total \\
\hline $\mathrm{C}_{1}$ & 0.57915 & 1.99937 & 5.56249 & 7.57915 \\
\hline $\mathrm{C}_{2}$ & 0.08908 & 1.99912 & 4.06298 & 6.08908 \\
\hline $\mathrm{C}_{3}$ & 0.04278 & 1.99910 & 4.02585 & 6.04278 \\
\hline $\mathrm{C}_{4}$ & 0.35421 & 1.99894 & 4.32938 & 6.35421 \\
\hline $\mathrm{C}_{5}$ & 0.04278 & 1.99910 & 4.02585 & 6.04278 \\
\hline $\mathrm{C}_{6}$ & 0.08908 & 1.99912 & 4.06298 & 6.08908 \\
\hline $\mathrm{C}_{7}$ & 0.43370 & 0.00000 & 5.06333 & 0.56630 \\
\hline $\mathrm{C}_{8}$ & 0.22971 & 0.00000 & 0.76896 & 0.77029 \\
\hline $\mathrm{C}_{9}$ & 0.69612 & 1.99949 & 4.68856 & 6.69612 \\
\hline $\mathrm{H}_{10}$ & 0.24329 & 0.00000 & 0.75479 & 0.75671 \\
\hline $\mathrm{H}_{11}$ & 0.24028 & 0.00000 & 0.75813 & 0.75972 \\
\hline $\mathrm{H}_{12}$ & 0.24861 & 0.00000 & 0.75025 & 0.75139 \\
\hline $\mathrm{H}_{13}$ & 0.23186 & 0.00000 & 0.76657 & 0.76814 \\
\hline $\mathrm{C}_{14}$ & 0.69612 & 1.99949 & 4.68856 & 6.69612 \\
\hline $\mathrm{H}_{15}$ & 0.24329 & 0.00000 & 0.75479 & 0.75671 \\
\hline $\mathrm{H}_{16}$ & 0.24861 & 0.00000 & 0.75025 & 0.75139 \\
\hline $\mathrm{N}_{17}$ & 0.24028 & 0.00000 & 0.75813 & 0.75972 \\
\hline $\mathrm{H}_{18}$ & 0.22971 & 0.00000 & 0.76896 & 0.77029 \\
\hline $\mathrm{C}_{19}$ & 1.99918 & 3.85572 & 5.87797 & 0.12203 \\
\hline $\mathrm{C}_{20}$ & 1.99908 & 4.24143 & 6.25510 & 0.25510 \\
\hline $\mathrm{N}_{21}$ & 1.99917 & 4.32244 & 6.34987 & 0.34987 \\
\hline $\mathrm{C}_{22}$ & 1.99907 & 4.25893 & 6.27356 & 0.27356 \\
\hline $\mathrm{C}_{23}$ & 1.99918 & 3.81252 & 5.83489 & 0.16511 \\
\hline $\mathrm{C}_{24}$ & 1.99970 & 5.68029 & 7.70435 & 0.70435 \\
\hline $\mathrm{C}_{25}$ & 0.00000 & 0.60930 & 0.61390 & 0.38610 \\
\hline $\mathrm{O}_{26}$ & 0.00000 & 0.59999 & 0.60222 & 0.39778 \\
\hline $\mathrm{H}_{27}$ & 1.99947 & 4.68963 & 6.69796 & 0.69796 \\
\hline $\mathrm{N}_{28}$ & 0.00000 & 0.74071 & 0.74184 & 0.25816 \\
\hline $\mathrm{H}_{29}$ & 0.00000 & 0.75060 & 0.75244 & 0.24756 \\
\hline $\mathrm{O}_{30}$ & 0.00000 & 0.78330 & 0.78514 & 0.21486 \\
\hline $\mathrm{N}_{31}$ & 0.00000 & 0.75707 & 0.75856 & 0.14424 \\
\hline $\mathrm{H}_{32}$ & 0.00000 & 0.76448 & 0.76614 & 0.38623 \\
\hline $\mathrm{O}_{33}$ & 0.00000 & 0.75516 & 0.75663 & 0.27433 \\
\hline $\mathrm{C}_{34}$ & 1.99948 & 4.70428 & 6.71144 & 0.74114 \\
\hline $\mathrm{C}_{35}$ & 0.00000 & 0.74098 & 0.75099 & 0.21490 \\
\hline $\mathrm{C}_{36}$ & 0.00000 & 0.75376 & 0.73934 & 0.26606 \\
\hline $\mathrm{N}_{37}$ & 0.22840 & 0.00054 & 0.73581 & 0.72597 \\
\hline $\mathrm{O}_{38}$ & 0.21297 & 0.00076 & 0.76689 & 0.77902 \\
\hline $\mathrm{O}_{39}$ & 1.98991 & 3.85572 & 5.87779 & 0.13220 \\
\hline $\mathrm{O}_{40}$ & 1.98990 & 4.24143 & 6.20551 & 0.20551 \\
\hline
\end{tabular}


Table 4. The atomic orbital occupancies of LAP.

\begin{tabular}{|c|c|c|c|c|}
\hline Atom No. & Atomic orbital & Type & Occupancy & Energy \\
\hline \multirow{5}{*}{$\mathrm{C}_{1}$} & $1 \mathrm{~s}$ & Core & 0.99969 & 14.19257 \\
\hline & $2 \mathrm{~s}$ & \multirow{4}{*}{ Valence } & 0.61019 & 0.52846 \\
\hline & $2 p_{x}$ & & 0.93370 & 0.28160 \\
\hline & $2 p_{y}$ & & 0.70719 & 0.25367 \\
\hline & $2 \mathrm{pz}$ & & 0.65583 & 0.24434 \\
\hline \multirow{5}{*}{$\mathrm{C}_{2}$} & $1 \mathrm{~s}$ & Core & 0.99956 & 10.06820 \\
\hline & $2 \mathrm{~s}$ & \multirow{4}{*}{ Valence } & 0.45872 & 0.16227 \\
\hline & $2 p_{x}$ & & 0.66749 & 0.13422 \\
\hline & $2 p_{y}$ & & 0.52767 & 0.03912 \\
\hline & $2 \mathrm{pz}$ & & 0.49784 & 0.02260 \\
\hline \multirow{5}{*}{$\mathrm{C}_{3}$} & $1 \mathrm{~s}$ & Core & 0.99955 & 10.04104 \\
\hline & $2 \mathrm{~s}$ & \multirow{4}{*}{ Valence } & 0.44940 & 0.14922 \\
\hline & $2 p_{x}$ & & 0.46622 & 0.07889 \\
\hline & $2 p_{y}$ & & 0.53180 & 0.03465 \\
\hline & $2 \mathrm{pz}$ & & 0.52968 & 0.03196 \\
\hline \multirow{5}{*}{$\mathrm{C}_{4}$} & $1 \mathrm{~s}$ & Core & 0.99948 & 9.99978 \\
\hline & $2 \mathrm{~s}$ & \multirow{4}{*}{ Valence } & 0.47403 & 0.13927 \\
\hline & $2 p_{x}$ & & 0.76074 & 0.12199 \\
\hline & $2 p_{y}$ & & 0.60256 & 0.03736 \\
\hline & $2 \mathrm{pz}$ & & 0.52857 & 0.02050 \\
\hline \multirow{5}{*}{$\mathrm{C}_{5}$} & $1 \mathrm{~s}$ & Core & 0.99955 & 10.04104 \\
\hline & $2 \mathrm{~s}$ & \multirow{4}{*}{ Valence } & 0.44940 & 0.14922 \\
\hline & $2 p_{x}$ & & 0.46622 & 0.07889 \\
\hline & $2 p_{y}$ & & 0.53180 & 0.03465 \\
\hline & $2 \mathrm{pz}$ & & 0.52968 & 0.03196 \\
\hline \multirow{5}{*}{$\mathrm{C}_{6}$} & $1 \mathrm{~s}$ & Core & 0.99956 & 10.06820 \\
\hline & $2 \mathrm{~s}$ & \multirow{4}{*}{ Valence } & 0.45872 & 0.16227 \\
\hline & $2 p_{x}$ & & 0.66749 & 0.13422 \\
\hline & $2 p_{y}$ & & 0.52767 & 0.03912 \\
\hline & $2 \mathrm{pz}$ & & 0.49784 & 0.02260 \\
\hline $\mathrm{C}_{7}$ & $1 \mathrm{~s}$ & & 0.27777 & 0.11773 \\
\hline $\mathrm{C}_{8}$ & $1 \mathrm{~s}$ & & 0.38069 & 0.08826 \\
\hline \multirow{5}{*}{$\mathrm{C}_{9}$} & $1 \mathrm{~s}$ & Core & 0.99974 & 10.03765 \\
\hline & $2 \mathrm{~s}$ & \multirow{7}{*}{ Valence } & 0.54920 & 0.24879 \\
\hline & $2 p_{x}$ & & 0.62806 & 0.07543 \\
\hline & $2 p_{y}$ & & 0.60980 & 0.07456 \\
\hline & $2 \mathrm{pz}$ & & 0.55944 & 0.06806 \\
\hline $\mathrm{H}_{10}$ & $1 \mathrm{~s}$ & & 0.37769 & 0.10148 \\
\hline $\mathrm{H}_{11}$ & $1 \mathrm{~s}$ & & 0.37838 & 0.09800 \\
\hline $\mathrm{H}_{12}$ & $1 \mathrm{~s}$ & & 0.37403 & 0.10171 \\
\hline $\mathrm{H}_{13}$ & $1 \mathrm{~s}$ & & 0.37728 & 0.11764 \\
\hline \multirow{4}{*}{$\mathrm{C}_{14}$} & $1 \mathrm{~s}$ & Core & 0.99974 & 10.03765 \\
\hline & $2 \mathrm{~s}$ & \multirow{3}{*}{ Valence } & 0.54920 & 0.24879 \\
\hline & $2 p_{x}$ & & 0.62806 & 0.07543 \\
\hline & $2 p_{y}$ & & 0.60980 & 0.07456 \\
\hline & $1 \mathrm{~s}$ & Core & 0.99953 & -14.21383 \\
\hline & $2 \mathrm{~s}$ & & 0.56716 & -0.49330 \\
\hline $\mathrm{N}_{17}$ & $2 p_{x}$ & Volence & 0.67535 & -0.27035 \\
\hline & $2 p_{y}$ & Valence & 0.57533 & -0.23914 \\
\hline & $2 \mathrm{pz}$ & & .92118 & -0.28706 \\
\hline & $1 \mathrm{~s}$ & Core & 0.99959 & -10.07773 \\
\hline & $2 \mathrm{~s}$ & & 0.42980 & -0.15686 \\
\hline $\mathrm{N}_{21}$ & $2 p_{x}$ & Valence & 0.44890 & -0.01282 \\
\hline & $2 p_{y}$ & Valence & 0.51352 & -0.03957 \\
\hline & $2 \mathrm{pz}$ & & 0.66295 & -0.13219 \\
\hline & $1 \mathrm{~s}$ & Core & 0.99954 & -10.02546 \\
\hline & $2 \mathrm{~s}$ & & 0.47522 & -0.15235 \\
\hline $\mathrm{N}_{25}$ & $2 p_{x}$ & & 0.58443 & -0.03429 \\
\hline & $2 p_{y}$ & Valence & 0.53812 & -0.02757 \\
\hline & $2 \mathrm{pz}$ & & 0.47394 & -0.08382 \\
\hline
\end{tabular}




\begin{tabular}{|c|c|c|c|c|}
\hline Atom No. & Atomic orbital & Type & Occupancy & Energy \\
\hline \multirow{5}{*}{$\mathrm{N}_{28}$} & $1 \mathrm{~s}$ & Core & 0.99959 & -10.00565 \\
\hline & $2 \mathrm{~s}$ & \multirow{4}{*}{ Valence } & 0.48238 & -0.15531 \\
\hline & $2 p_{x}$ & & 0.52473 & -0.01345 \\
\hline & $2 p_{y}$ & & 0.60187 & -0.02854 \\
\hline & $2 \mathrm{pz}$ & & 0.76549 & -0.11905 \\
\hline \multirow{5}{*}{$\mathrm{O}_{26}$} & $1 \mathrm{~s}$ & Core & 0.99954 & -10.02154 \\
\hline & $2 \mathrm{~s}$ & \multirow{4}{*}{ Valence } & 0.47599 & -0.15082 \\
\hline & $2 p_{x}$ & & 0.59004 & -0.03438 \\
\hline & $2 p_{y}$ & & 0.53285 & -0.02489 \\
\hline & $2 \mathrm{pz}$ & & 0.50166 & -0.08534 \\
\hline \multirow{5}{*}{$\mathrm{N}_{31}$} & $1 \mathrm{~s}$ & Core & 0.99959 & -10.07826 \\
\hline & $2 \mathrm{~s}$ & \multirow{4}{*}{ Valence } & 0.43073 & -0.15225 \\
\hline & $2 p_{x}$ & & 0.44169 & -0.00290 \\
\hline & $2 p_{y}$ & & 0.51983 & -0.03708 \\
\hline & $2 \mathrm{pz}$ & & 0.61461 & -0.12140 \\
\hline \multirow{5}{*}{$\mathrm{N}_{37}$} & $1 \mathrm{~s}$ & Core & 0.99985 & -14.22801 \\
\hline & $2 \mathrm{~s}$ & \multirow{6}{*}{ Valence } & 0.70247 & -0.57543 \\
\hline & $2 p_{x}$ & & 0.85160 & -0.25208 \\
\hline & $2 p_{y}$ & & 0.54018 & -0.17893 \\
\hline & $2 \mathrm{pz}$ & & 0.74219 & -0.22576 \\
\hline $\mathrm{H}_{22}$ & $1 \mathrm{~s}$ & & 0.31341 & 0.11748 \\
\hline $\mathrm{H}_{27}$ & $1 \mathrm{~s}$ & & 0.30096 & 0.13522 \\
\hline \multirow{5}{*}{$\mathrm{O}_{30}$} & $1 \mathrm{~s}$ & Core & 0.99973 & -10.05781 \\
\hline & $2 \mathrm{~s}$ & \multirow{5}{*}{ Valence } & 0.54819 & -0.26511 \\
\hline & $2 p_{x}$ & & 0.56106 & -0.09156 \\
\hline & $2 p_{y}$ & & 0.60209 & -0.09170 \\
\hline & $2 \mathrm{pz}$ & & 0.62649 & -0.09324 \\
\hline $\mathrm{H}_{29}$ & $1 \mathrm{~s}$ & & 0.37168 & 0.09545 \\
\hline \multirow[t]{5}{*}{$\mathrm{O}_{33}$} & $1 \mathrm{~s}$ & Core & 0.99599 & -10.07682 \\
\hline & $2 \mathrm{~s}$ & \multirow{4}{*}{ Valence } & 0.43307 & -0.15522 \\
\hline & $2 \mathrm{px}$ & & 0.44916 & -0.00029 \\
\hline & 2 py & & 0.53198 & -0.00837 \\
\hline & $2 \mathrm{pz}$ & & 0.61146 & -0.14021 \\
\hline \multirow[t]{5}{*}{$\mathrm{O}_{38}$} & $1 \mathrm{~s}$ & Core & 0.95998 & -14.21280 \\
\hline & $2 \mathrm{~s}$ & \multirow{4}{*}{ Valence } & 0.77024 & -0.53754 \\
\hline & $2 \mathrm{px}$ & & 0.80516 & -0.28520 \\
\hline & $2 p y$ & & 0.58401 & -0.13789 \\
\hline & $2 \mathrm{pz}$ & & 0.79421 & -0.26257 \\
\hline
\end{tabular}

Table 5. Bond orbital analysis of LAP by B3LYP/6-31+G(d,p) method and basis set.

\begin{tabular}{|c|c|c|c|c|c|}
\hline Bond orbital & Occupancy & Atom & $\begin{array}{c}\text { Contribution } \\
\text { from parent } \\
\text { NBO }(\%)\end{array}$ & Coefficients & Atom Hybrid Contributions (\%) \\
\hline \multirow{2}{*}{$\mathrm{C}_{1}-\mathrm{C}_{2}$} & \multirow{2}{*}{0.99284} & $\mathrm{~N}_{1}$ & 62.74 & 0.7921 & $\mathrm{~s}(36.47)+\mathrm{p} 1.74(63.50)$ \\
\hline & & $\mathrm{C}_{2}$ & 37.26 & 0.6104 & $\mathrm{~s}(29.37)+\mathrm{p} 2.40(70.51)$ \\
\hline \multirow{2}{*}{$\mathrm{C}_{1}-\mathrm{C}_{6}$} & \multirow{2}{*}{0.99284} & $\mathrm{~N}_{1}$ & 62.74 & 0.7921 & $s(36.47)+p 1.74(63.50)$ \\
\hline & & $\mathrm{C}_{6}$ & 37.26 & 0.6104 & $\mathrm{~s}(29.37)+\mathrm{p} 2.40(70.51)$ \\
\hline \multirow{2}{*}{$\mathrm{N}_{17}-\mathrm{C}_{7}$} & \multirow{2}{*}{0.99280} & $\mathrm{~N}_{1}$ & 72.56 & 0.8518 & $\mathrm{~s}(26.98)+\mathrm{p} 2.71(73.00)$ \\
\hline & & $\mathrm{H}_{7}$ & 27.44 & 0.5238 & $\mathrm{~s}(99.89)+\mathrm{p} 0.00(0.11)$ \\
\hline \multirow{2}{*}{$\mathrm{C}_{2}-\mathrm{C}_{3}$} & \multirow{2}{*}{0.98823} & $\mathrm{C}_{2}$ & 50.00 & 0.7071 & $\mathrm{~s}(37.47)+\mathrm{p} 1.67(62.50)$ \\
\hline & & $\mathrm{C}_{3}$ & 50.00 & 0.7071 & $\mathrm{~s}(33.15)+\mathrm{p} 2.02(66.81)$ \\
\hline \multirow{2}{*}{$\mathrm{C}_{2}-\mathrm{H}_{10}$} & \multirow{2}{*}{0.99088} & $\mathrm{C}_{2}$ & 61.90 & 0.7868 & $\mathrm{~s}(32.59)+\mathrm{p} 2.07(67.37)$ \\
\hline & & $\mathrm{H}_{8}$ & 38.10 & 0.6172 & $\mathrm{~s}(99.95)+\mathrm{p} 0.00(0.05)$ \\
\hline \multirow{2}{*}{$\mathrm{C}_{3}-\mathrm{C}_{4}$} & \multirow{2}{*}{0.98751} & $\mathrm{C}_{2}$ & 50.48 & 0.7105 & $\mathrm{~s}(35.17)+\mathrm{p} 1.84(64.79)$ \\
\hline & & $\mathrm{C}_{4}$ & 49.52 & 0.7037 & $\mathrm{~s}(34.46)+\mathrm{p} 1.90(65.51)$ \\
\hline \multirow{2}{*}{$\mathrm{C}_{3}-\mathrm{C}_{9}$} & \multirow{2}{*}{0.99160} & $\mathrm{C}_{3}$ & 50.88 & 0.7133 & $\mathrm{~s}(31.13)+\mathrm{p} 2.21(68.84)$ \\
\hline & & $\mathrm{C}_{9}$ & 49.12 & 0.7009 & $\mathrm{~s}(25.62)+\mathrm{p} 2.90(74.34)$ \\
\hline \multirow{2}{*}{$\mathrm{C}_{4}-\mathrm{C}_{5}$} & \multirow{2}{*}{0.98751} & $\mathrm{C}_{4}$ & 49.52 & 0.7037 & $\mathrm{~s}(34.46)+\mathrm{p} 1.90(65.51)$ \\
\hline & & $\mathrm{C}_{5}$ & 50.48 & 0.7105 & $\mathrm{~s}(35.17)+\mathrm{p} 1.84(64.79)$ \\
\hline \multirow{2}{*}{$\mathrm{C}_{4}-\mathrm{H}_{13}$} & \multirow{2}{*}{0.98995} & $\mathrm{C}_{4}$ & 62.31 & 0.7893 & $\mathrm{~s}(31.07)+\mathrm{p} 2.22(68.90)$ \\
\hline & & $\mathrm{H}_{13}$ & 37.69 & 0.6140 & $\mathrm{~s}(99.95)+\mathrm{p} 0.00(0.05)$ \\
\hline \multirow{2}{*}{$\mathrm{C}_{5}-\mathrm{C}_{6}$} & \multirow{2}{*}{0.98823} & $\mathrm{C}_{5}$ & 50.00 & 0.7071 & $\mathrm{~s}(33.15)+\mathrm{p} 2.02(66.81)$ \\
\hline & & $\mathrm{C}_{6}$ & 50.00 & 0.7071 & $\mathrm{~s}(37.47)+\mathrm{p} 1.67(62.50)$ \\
\hline
\end{tabular}


https://doi.org/10.33263/BRIAC126.83538366

\begin{tabular}{|c|c|c|c|c|c|}
\hline Bond orbital & Occupancy & Atom & $\begin{array}{c}\text { Contribution } \\
\text { from parent } \\
\text { NBO }(\%) \\
\end{array}$ & Coefficients & Atom Hybrid Contributions (\%) \\
\hline \multirow{2}{*}{$\mathrm{C}_{5}-\mathrm{H}_{14}$} & \multirow{2}{*}{0.99160} & $\mathrm{C}_{5}$ & 50.88 & 0.7133 & $\mathrm{~s}(31.13)+\mathrm{p} 2.21(68.84)$ \\
\hline & & $\mathrm{C}_{14}$ & 49.12 & 0.7009 & $\mathrm{~s}(25.62)+\mathrm{p} 2.90(74.34)$ \\
\hline \multirow{2}{*}{$\mathrm{C}_{6}-\mathrm{H}_{16}$} & \multirow{2}{*}{0.99088} & $\mathrm{C}_{6}$ & 61.90 & 0.7868 & $\mathrm{~s}(32.59)+\mathrm{p} 2.07(67.37)$ \\
\hline & & $\mathrm{H}_{18}$ & 38.10 & 0.6172 & $\mathrm{~s}(99.95)+\mathrm{p} 0.00(0.05)$ \\
\hline \multirow{2}{*}{$\mathrm{C}_{9}-\mathrm{H}_{10}$} & \multirow{2}{*}{0.99388} & $\mathrm{C}_{9}$ & 62.24 & 0.7889 & $\mathrm{~s}(25.05)+\mathrm{p} 2.99(74.91)$ \\
\hline & & $\mathrm{H}_{10}$ & 37.76 & 0.6145 & $\mathrm{~s}(99.95)+\mathrm{p} 0.00(0.05)$ \\
\hline \multirow{2}{*}{$\mathrm{C}_{9}-\mathrm{H}_{11}$} & \multirow{2}{*}{0.99433} & $\mathrm{C}_{9}$ & 62.08 & 0.7879 & $\mathrm{~s}(24.71)+\mathrm{p} 3.04(75.24)$ \\
\hline & & $\mathrm{H}_{11}$ & 37.92 & 0.6158 & $\mathrm{~s}(99.95)+\mathrm{p} 0.00(0.05)$ \\
\hline \multirow{2}{*}{$\mathrm{C}_{9}-\mathrm{H}_{12}$} & \multirow{2}{*}{0.98965} & $\mathrm{C}_{9}$ & 62.43 & 0.7901 & $\mathrm{~s}(24.59)+\mathrm{p} 3.06(75.36)$ \\
\hline & & $\mathrm{H}_{12}$ & 37.57 & 0.6129 & $\mathrm{~s}(99.95)+\mathrm{p} 0.00(0.05)$ \\
\hline \multirow{2}{*}{$\mathrm{H}_{14}-\mathrm{H}_{27}$} & \multirow{2}{*}{0.99388} & $\mathrm{C}_{14}$ & 62.24 & 0.7889 & $\mathrm{~s}(25.05)+\mathrm{p} 2.99(74.91)$ \\
\hline & & $\mathrm{H}_{15}$ & 37.76 & 0.6145 & $\mathrm{~s}(99.95)+\mathrm{p} 0.00(0.05)$ \\
\hline \multirow{2}{*}{$\mathrm{H}_{14}-\mathrm{H}_{16}$} & \multirow{2}{*}{0.98965} & $\mathrm{C}_{14}$ & 62.43 & 0.7901 & $\mathrm{~s}(24.59)+\mathrm{p} 3.06(75.36)$ \\
\hline & & $\mathrm{H}_{16}$ & 37.57 & 0.6129 & $\mathrm{~s}(99.95)+\mathrm{p} 0.00(0.05)$ \\
\hline \multirow[b]{2}{*}{$\mathrm{H}_{14}-\mathrm{H}_{29}$} & \multirow{2}{*}{0.99433} & $\mathrm{C}_{14}$ & 62.08 & 0.7879 & $\mathrm{~s}(24.71)+\mathrm{p} 3.04(75.24)$ \\
\hline & & $\mathrm{H}_{17}$ & 37.92 & 0.6158 & $\mathrm{~s}(99.95)+\mathrm{p} 0.00(0.05)$ \\
\hline \multirow{2}{*}{$\mathrm{N}_{21}-\mathrm{C}_{7}$} & \multirow{2}{*}{0.99109} & $\mathrm{~N}_{1}$ & 63.50 & 0.7969 & $\mathrm{~s}(37.00)+\mathrm{p} 1.70(62.98)$ \\
\hline & & $\mathrm{C}_{2}$ & 36.50 & 0.6042 & $\mathrm{~s}(28.65)+\mathrm{p} 2.49(71.23)$ \\
\hline \multirow{2}{*}{$\mathrm{N}_{28}-\mathrm{H}_{16}$} & \multirow{2}{*}{0.99026} & $\mathrm{~N}_{1}$ & 63.89 & 0.7993 & $\mathrm{~s}(37.56)+\mathrm{p} 1.66(62.42)$ \\
\hline & & $\mathrm{C}_{6}$ & 36.11 & 0.6009 & $\mathrm{~s}(28.46)+\mathrm{p} 2.51(71.42)$ \\
\hline \multirow{2}{*}{$\mathrm{N}_{31}-\mathrm{N}_{37}$} & \multirow{2}{*}{0.99491} & $\mathrm{~N}_{1}$ & 54.08 & 0.7354 & $\mathrm{~s}(25.36)+\mathrm{p} 2.94(74.59)$ \\
\hline & & $\mathrm{N}_{7}$ & 45.92 & 0.6776 & $\mathrm{~s}(24.16)+\mathrm{p} 3.13(75.72)$ \\
\hline \multirow{2}{*}{$\mathrm{H}_{12}-\mathrm{H}_{13}$} & \multirow{2}{*}{0.98692} & $\mathrm{C}_{2}$ & 51.53 & 0.7179 & $\mathrm{~s}(38.69)+\mathrm{p} 1.58(61.28)$ \\
\hline & & $\mathrm{C}_{3}$ & 48.47 & 0.6962 & $\mathrm{~s}(33.34)+\mathrm{p} 2.00(66.61)$ \\
\hline \multirow{2}{*}{$\mathrm{H}_{14}-\mathrm{O}_{33}$} & \multirow{2}{*}{0.99155} & $\mathrm{C}_{2}$ & 50.82 & 0.7129 & $\mathrm{~s}(32.61)+\mathrm{p} 2.07(67.37)$ \\
\hline & & $\mathrm{C}_{10}$ & 49.18 & 0.7013 & $\mathrm{~s}(24.68)+\mathrm{p} 3.05(75.27)$ \\
\hline & & $\mathrm{C}_{3}$ & 50.32 & 0.7094 & $\mathrm{~s}(35.23)+\mathrm{p} 1.84(64.73)$ \\
\hline $\mathrm{O}_{33}-\mathrm{H}_{27}$ & 0.98920 & $\mathrm{C}_{4}$ & 49.68 & 0.7049 & $\mathrm{~s}(34.52)+\mathrm{p} 1.90(65.44)$ \\
\hline & & $\mathrm{C}_{3}$ & 61.96 & 0.7871 & $\mathrm{~s}(31.35)+\mathrm{p} 2.19(68.61)$ \\
\hline $\mathrm{O}_{33}-\mathrm{H}_{30}$ & 0.98981 & $\mathrm{H}_{14}$ & 38.04 & 0.6168 & $\mathrm{~s}(99.95)+\mathrm{p} 0.00(0.05)$ \\
\hline $\mathrm{C}_{4}-\mathrm{H}_{4}$ & 008020 & $\mathrm{C}_{4}$ & 49.79 & 0.7056 & $\mathrm{~s}(34.61)+\mathrm{p} 1.89(65.36)$ \\
\hline $\mathrm{C}_{44}-\mathrm{H}_{47}$ & 0.98929 & $\mathrm{C}_{5}$ & 50.21 & 0.7086 & $\mathrm{~s}(35.06)+\mathrm{p} 1.85(64.90)$ \\
\hline & & $\mathrm{C}_{4}$ & 62.44 & 0.7902 & $\mathrm{~s}(30.86)+\mathrm{p} 2.24(69.11)$ \\
\hline $\mathrm{O}_{38}-\mathrm{H}_{18}$ & 0.99113 & $\mathrm{H}_{15}$ & 37.56 & 0.6129 & $\mathrm{~s}(99.95)+\mathrm{p} 0.00(0.05)$ \\
\hline & & $\mathrm{C}_{5}$ & 48.59 & 0.6970 & $\mathrm{~s}(33.37)+\mathrm{p} 2.00(66.59)$ \\
\hline $\mathrm{C}_{25}-\mathrm{C}_{22}$ & 0.98692 & $\mathrm{C}_{6}$ & 51.41 & 0.7170 & $\mathrm{~s}(38.58)+\mathrm{p} 1.59(61.39)$ \\
\hline $\mathrm{H}_{2}-\mathrm{H}$ & & $\mathrm{C}_{5}$ & 62.10 & 0.7880 & $\mathrm{~s}(31.50)+\mathrm{p} 2.17(68.46)$ \\
\hline $\mathrm{H}_{27}-\mathrm{H}_{29}$ & 0.98967 & $\mathrm{H}_{16}$ & 37.90 & 0.6156 & $\mathrm{~s}(99.95)+\mathrm{p} 0.00(0.05)$ \\
\hline & & $\mathrm{C}_{6}$ & 51.52 & 0.7178 & $\mathrm{~s}(32.94)+\mathrm{p} 2.03(67.03)$ \\
\hline $\mathrm{O}_{38}-\mathrm{H}_{47}$ & 0.99100 & $\mathrm{C}_{17}$ & 48.48 & 0.6963 & $\mathrm{~s}(24.60)+\mathrm{p} 3.06(75.35)$ \\
\hline
\end{tabular}

Table 6. Second-order perturbation analysis of Fock matrix in NBO basis for LAP.

\begin{tabular}{c|c|c|c} 
Donor(i) $\rightarrow$ Acceptor(j) & $\mathbf{E}(\mathbf{2})^{\mathbf{a}}$ & $\mathbf{E}(\mathbf{j})-\mathbf{E}(\mathbf{i})^{\mathbf{b}}$ & $\mathbf{F}(\mathbf{i}, \mathbf{j})^{\mathbf{c}}$ \\
\hline$\sigma \mathrm{C}_{2}-\mathrm{H}_{10} \rightarrow \sigma^{*} \mathrm{C}_{1}-\mathrm{C}_{6}$ & 2.21 & 1.06 & 0.061 \\
\hline$\sigma \mathrm{C}_{4}-\mathrm{H}_{13} \rightarrow \sigma^{*} \mathrm{C}_{2}-\mathrm{C}_{3}$ & 2.00 & 1.07 & 0.059 \\
\hline$\sigma \mathrm{C}_{4}-\mathrm{H}_{13} \rightarrow \sigma^{*} \mathrm{C}_{5}-\mathrm{C}_{6}$ & 2.00 & 1.07 & 0.059 \\
\hline$\sigma \mathrm{C}_{6}-\mathrm{H}_{18} \rightarrow \sigma^{*} \mathrm{~N}_{1}-\mathrm{C}_{2}$ & 2.21 & 1.06 & 0.061 \\
\hline$\pi \mathrm{C}_{1}-\mathrm{C}_{6} \rightarrow \pi^{*} \mathrm{C}_{2}-\mathrm{C}_{3}$ & 12.18 & 0.36 & 0.086 \\
\hline$\pi \mathrm{C}_{2}-\mathrm{C}_{3} \rightarrow \mathrm{n}^{*} \mathrm{C}_{4}$ & 27.34 & 0.18 & 0.100 \\
\hline$\pi \mathrm{C}_{2}-\mathrm{C}_{3} \rightarrow \pi^{*} \mathrm{~N}_{28}-\mathrm{C}_{6}$ & 6.86 & 0.23 & 0.052 \\
\hline$n \mathrm{C}_{5} \rightarrow \mathrm{n}^{*} \mathrm{C}_{34}$ & 270.93 & 0.14 & 0.141 \\
\hline$n \mathrm{C}_{36} \rightarrow \pi^{*} \mathrm{~N}_{31}-\mathrm{C}_{36}$ & 74.83 & 0.09 & 0.121 \\
\hline$n \mathrm{C}_{34} \rightarrow \sigma * \mathrm{C}_{7}-\mathrm{H}_{17}$ & 2.23 & 0.58 & 0.062 \\
\hline $\mathrm{n}^{*} \mathrm{C}_{34} \rightarrow \pi^{*} \mathrm{C}_{2}-\mathrm{C}_{3}$ & 34.65 & 0.10 & 0.100 \\
\hline$\pi^{*} \mathrm{~N}_{31}-\mathrm{C}_{6} \rightarrow \pi^{*} \mathrm{C}_{2}-\mathrm{C}_{3}$ & 26.77 & 0.05 & 0.072 \\
\hline$\sigma \mathrm{N}_{37}-\mathrm{C}_{2} \rightarrow \sigma^{*} \mathrm{~N}_{21}-\mathrm{C}_{6}$ & 2.24 & 1.36 & 0.070 \\
\hline$\sigma \mathrm{N}_{28}-\mathrm{C}_{6} \rightarrow \sigma^{*} \mathrm{~N}_{37}-\mathrm{C}_{2}$ & 2.29 & 1.37 & 0.071 \\
\hline$\sigma \mathrm{C}_{2}-\mathrm{C}_{3} \rightarrow \sigma^{*} \mathrm{~N}_{21}-\mathrm{N}_{37}$ & 2.41 & 0.98 & 0.061 \\
\hline$\sigma \mathrm{C}_{2}-\mathrm{H}_{10} \rightarrow \sigma^{*} \mathrm{~N}_{28}-\mathrm{C}_{6}$ & 2.03 & 1.12 & 0.060 \\
\hline$\sigma \mathrm{C}_{5}-\mathrm{H}_{16} \rightarrow \sigma^{*} \mathrm{~N}_{31}-\mathrm{C}_{6}$ & 2.53 & 1.04 & 0.065 \\
\hline$\sigma \mathrm{C}_{17}-\mathrm{H}_{20} \rightarrow \sigma^{*} \mathrm{~N}_{21}-\mathrm{H}_{9}$ & 2.51 & 1.04 & 0.065 \\
\hline$\pi \mathrm{C}_{1}-\mathrm{C}_{2} \rightarrow \pi^{*} \mathrm{C}_{5}-\mathrm{C}_{6}$ & 14.04 & 0.37 & 0.092
\end{tabular}




\begin{tabular}{c|c|c|c} 
Donor $(\mathbf{i}) \rightarrow \operatorname{Acceptor}(\mathbf{j})$ & $\mathbf{E}(\mathbf{2})^{\mathbf{a}}$ & $\mathbf{E}(\mathbf{j})-\mathbf{E}(\mathbf{i})^{\mathbf{b}}$ & $\mathbf{F}(\mathbf{i}, \mathbf{j})^{\mathbf{c}}$ \\
\hline$\pi \mathrm{C}_{15}-\mathrm{C}_{6} \rightarrow \mathrm{n}^{*} \mathrm{C}_{44}$ & 26.71 & 0.19 & 0.101 \\
\hline$\pi \mathrm{C}_{5}-\mathrm{C}_{6} \rightarrow \pi^{*} \mathrm{~N}_{21}-\mathrm{C}_{2}$ & 5.47 & 0.24 & 0.048 \\
\hline $\mathrm{n}_{1} \mathrm{O}_{33} \rightarrow \mathrm{n}^{*} \mathrm{~N}_{37}$ & 207.43 & 0.05 & 0.140 \\
\hline $\mathrm{n}_{2} \mathrm{O}_{33} \rightarrow \pi^{*} \mathrm{~N}_{1}-\mathrm{C}_{2}$ & 71.75 & 0.10 & 0.124 \\
\hline $\mathrm{n}_{1} \mathrm{~N}_{31} \rightarrow \pi^{*} \mathrm{~N}_{1}-\mathrm{C}_{2}$ & 2.06 & 0.35 & 0.038 \\
\hline $\mathrm{n}_{2} \mathrm{~N}_{3} \rightarrow \sigma * \mathrm{~N}_{1}-\mathrm{C}_{6}$ & 1.98 & 0.86 & 0.052 \\
\hline $\mathrm{n}^{*} \mathrm{O}_{38} \rightarrow \pi^{*} \mathrm{C}_{5}-\mathrm{C}_{6}$ & 33.23 & 0.09 & 0.094 \\
\hline$\pi * \mathrm{C}_{1}-\mathrm{C}_{2} \rightarrow \pi^{*} \mathrm{C}_{5}-\mathrm{C}_{6}$ & 35.03 & 0.04 & 0.073
\end{tabular}

${ }^{b}$ Energy difference between donor and acceptor $i$ and $j$ NBO orbitals.

${ }^{c} \mathrm{~F}(\mathrm{ij})$ - Fock matrix element between $\mathrm{i}$ and $\mathrm{j}$ NBO orbitals.

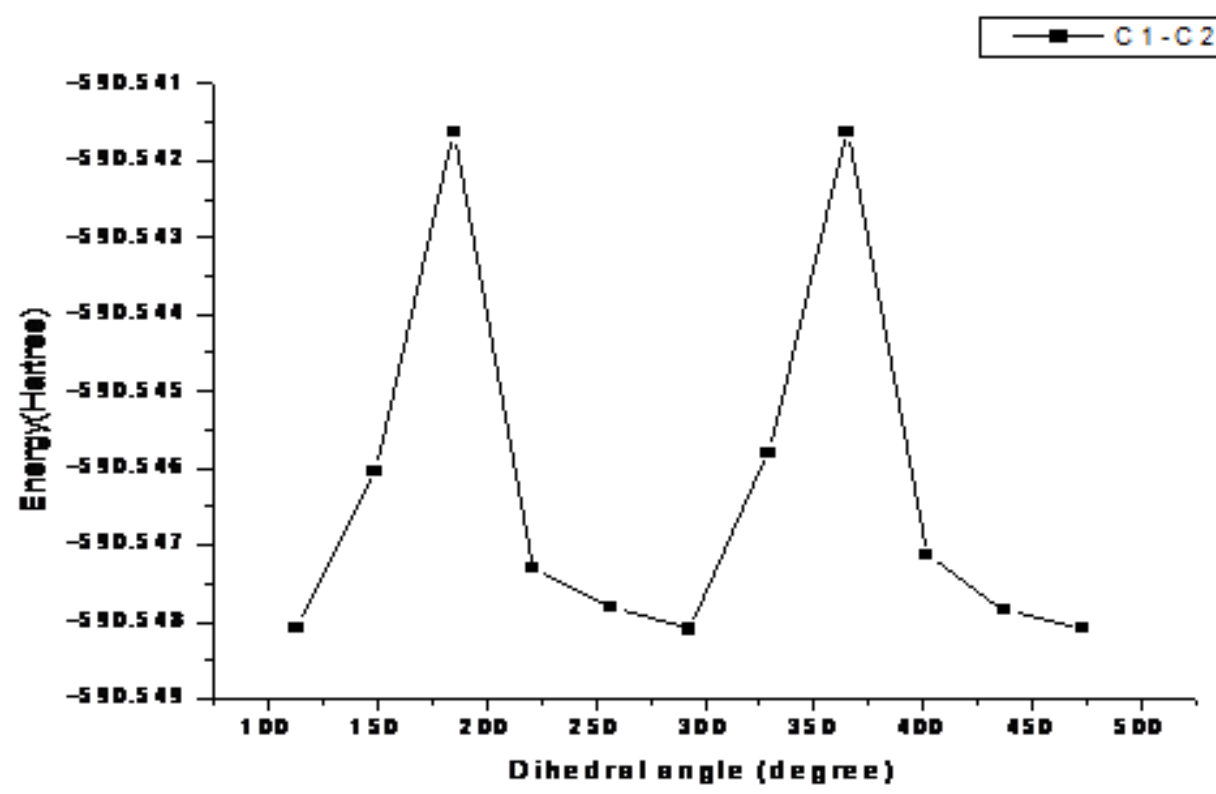

Figure 10. PES scan surface of LAP.

\subsection{PES scan studies.}

The potential energy surface (PES) scan for LAP was performed using the B3LYP/6$311+\mathrm{G}(\mathrm{d}, \mathrm{p})$ level of theoretical approximations, as shown in Figure 2. For conformational flexibility within the molecule, the dihedral angles $\mathrm{C}_{5}-\mathrm{C}_{6}-\mathrm{O}_{38}-\mathrm{C}_{19}$ and $\mathrm{C}_{1}-\mathrm{C}_{2}-\mathrm{N}_{21}-\mathrm{O}_{38}$ for LAP are also important coordinates. During the computation, all geometrical parameters are relaxed at the same time, while the torsional angles of $\mathrm{C}_{5}-\mathrm{C}_{6}-\mathrm{O}_{38}-\mathrm{C}_{19}$ and $\mathrm{C}_{1}-\mathrm{C}_{2}-\mathrm{N}_{21}-\mathrm{O}_{38}$ is varied in increments of $10^{\circ}, 20^{\circ}, 30^{\circ}, 360^{\circ}$. For both molecules, the highest energy is acquired at $180^{\circ}$.

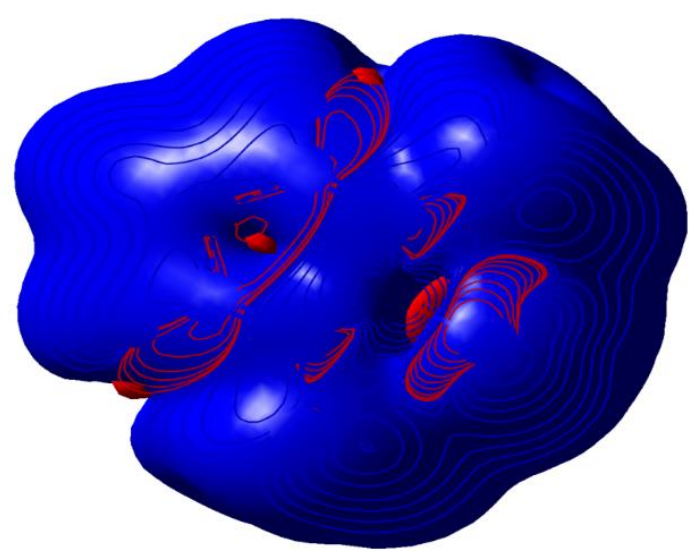

Figure3.NMR shielding surface of LAP. 


\section{NMR Spectral Analysis}

NMR Spectroscopy is a potent tool for deciphering the topology, dynamics, and threedimensional structure of molecules in solid-state and solution. Due to their chemical shift on the resonant frequencies of the nuclei present in the molecule, this approach is utilized to gain physical, chemical, electrical, and structural information about molecules. For calculating chemical shifts for various types of molecules, the GIAO/DFT (Gauge including atomic orbital/density functional theory) technique is extensively employed [22-24]. The chemical shift of a ${ }^{13} \mathrm{C}$ NMR atom in a specific place in a molecular system is an essential technique for determining that atom's presence or absence. Shielding of atoms (Figure 3) such as carbon in a molecule is highly influenced by nearby bound atoms, and similar bonded atoms have varying shielding values in different contexts. The chemical shift values for carbon atoms were investigated using the approved approach.

In the gas phase, the NMR calculations are performed at the B3LYP/6 - 31+G(d,p) levels of theory. Table 7 demonstrates that the typical organic molecule's range ${ }^{13} \mathrm{CNMR}$ chemical shift usually ensures reliable interpretation of spectroscopic parameters. So the $\mathrm{O}$ atoms have an electronegative property (they attract the other atom towards them), but the chemical shift of $\mathrm{C}_{2}, \mathrm{C}_{4}, \mathrm{C}_{6}$ appears to be $\left(\mathrm{C}_{2}=82.907 ; \mathrm{C}_{4}=92.800 ; \mathrm{C}_{6}=82.9 \mathrm{ppm}\right)$ by for LAP, but the chemical shift of N17 is higher, i.e., 153.765 and $135.62 \mathrm{ppm}$ by using B3LYP/6$31 \mathrm{G}+(\mathrm{d}, \mathrm{p})$ methods for LAP. The $\mathrm{H}$ atom is the tiniest of all atoms, and it is found predominantly on the edges of molecules. As a result, their chemical changes in aqueous solution would be more vulnerable to intermolecular interactions than those of other heavier atoms.

Table 7. Experimental and theoretical chemical shifts $\left({ }^{13} \mathrm{C},{ }^{1} \mathrm{H}\right)[\delta(\mathrm{ppm})]$ of LAP by B3LYP/6 $-31+\mathrm{G}(\mathrm{d}, \mathrm{p})$ methods and basis set.

\begin{tabular}{c|c|c|c}
\multirow{2}{*}{ Atom position } & \multicolumn{2}{|c|}{ B3LYP } & \multicolumn{1}{c}{ Expt } \\
\cline { 2 - 4 } & $\mathbf{6 - 3 1 + G ( d , p )}$ & & \\
\hline $\mathrm{C}_{1}$ & 153.765 & 111.02 & 28.113 \\
\hline $\mathrm{C}_{2}$ & 82.907 & 127.15 & 72.233 \\
\hline $\mathrm{C}_{3}$ & 54.917 & 127.15 & 35.68 \\
\hline $\mathrm{C}_{4}$ & 92.800 & 129.31 & 72.25 \\
\hline $\mathrm{C}_{5}$ & 54.9 & & 46.41 \\
\hline $\mathrm{C}_{6}$ & 82.9 & & \\
\hline $\mathrm{C}_{7}$ & 29.7 & & \\
\hline $\mathrm{C}_{8}$ & 27.1 & & \\
\hline $\mathrm{C}_{9}$ & 176.50 & 2.39 & \\
\hline $\mathrm{H}_{10}$ & 31.6 & 2.39 & \\
\hline $\mathrm{H}_{11}$ & 31.0 & 2.39 & \\
\hline $\mathrm{H}_{12}$ & 31.4 & & \\
\hline $\mathrm{H}_{13}$ & 28.5 & & \\
\hline $\mathrm{H}_{14}$ & 176.51 & & \\
\hline $\mathrm{H}_{15}$ & 31.6 & & \\
\hline $\mathrm{H}_{16}$ & 31.4 & & \\
\hline $\mathrm{N}_{17}$ & 31.0 & & \\
\hline $\mathrm{H}_{18}$ & 27.1 & & \\
\hline & & & \\
\hline & & & \\
\hline & & & \\
\hline & & & \\
\hline & & & \\
\hline & & & \\
\hline & & & \\
\hline
\end{tabular}

${ }^{\text {a }} \operatorname{Ref}[6]$. 


\section{Molecular Electronic Potential Maps}

The electrostatic potential is a three-dimensional indicator of molecular charge distribution. In fact, this image allows individuals to diagnose and analyze the charge distribution of atoms in different sections of the molecule. On the other hand, this map could be used to anticipate molecular behavior and performance in chemical reactions [24-26]. To generate and show this map, the corresponding structure was first optimized using density functional theory, and then the electrostatic potential map was calculated and recorded using the Molekel program. This map depicts the charge distribution mode for several sections of the molecule.

Figures 4-6 indicate the theoretical levels of B3LYP for electrophilic and nucleophilic processes. The lowest amount of electrostatic potential in this map is found in hydrogen atoms, while the highest level is found in oxygen atoms. Furthermore, the $\mathrm{O}$ atom has a green color and is in the middle of the electrostatic potential range. Furthermore, nitrogen atoms associated with the $\mathrm{N}$ group appear yellow in the electrostatic potential map and have a lower negative level than the $\mathrm{S}$ atom.

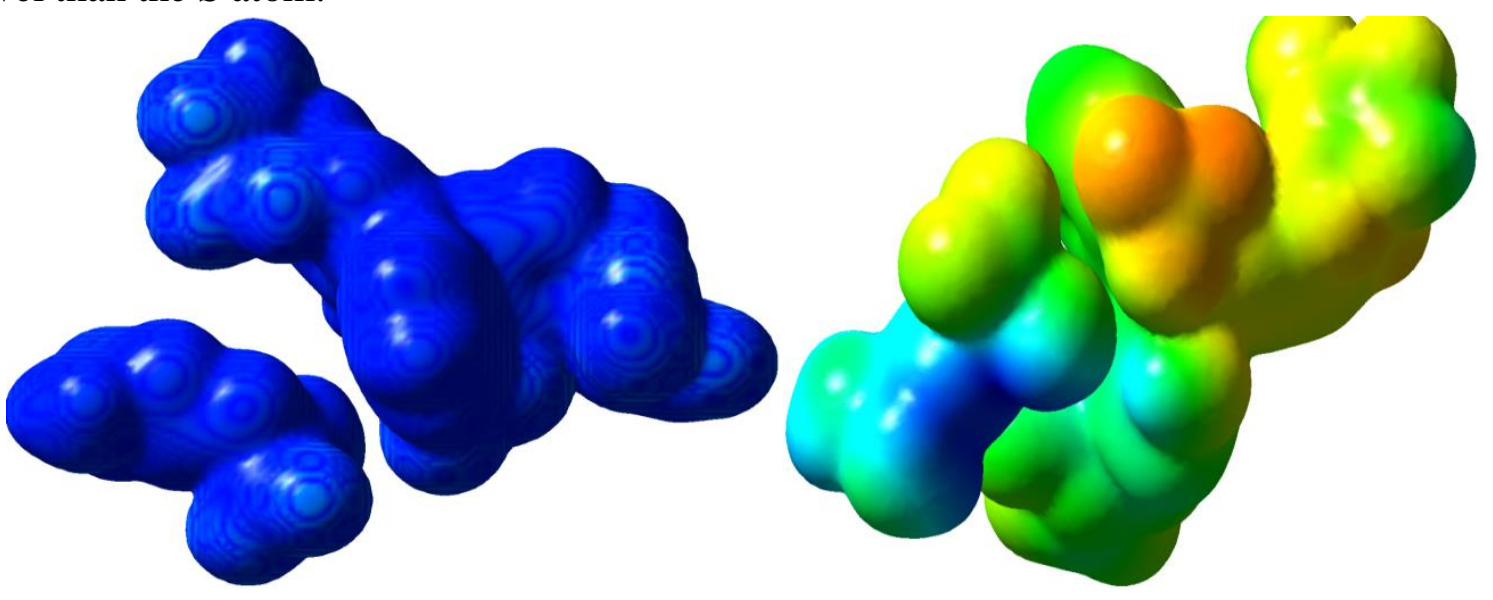

Figure 4. Different molecular electrostatic potential surfaces of LAP.
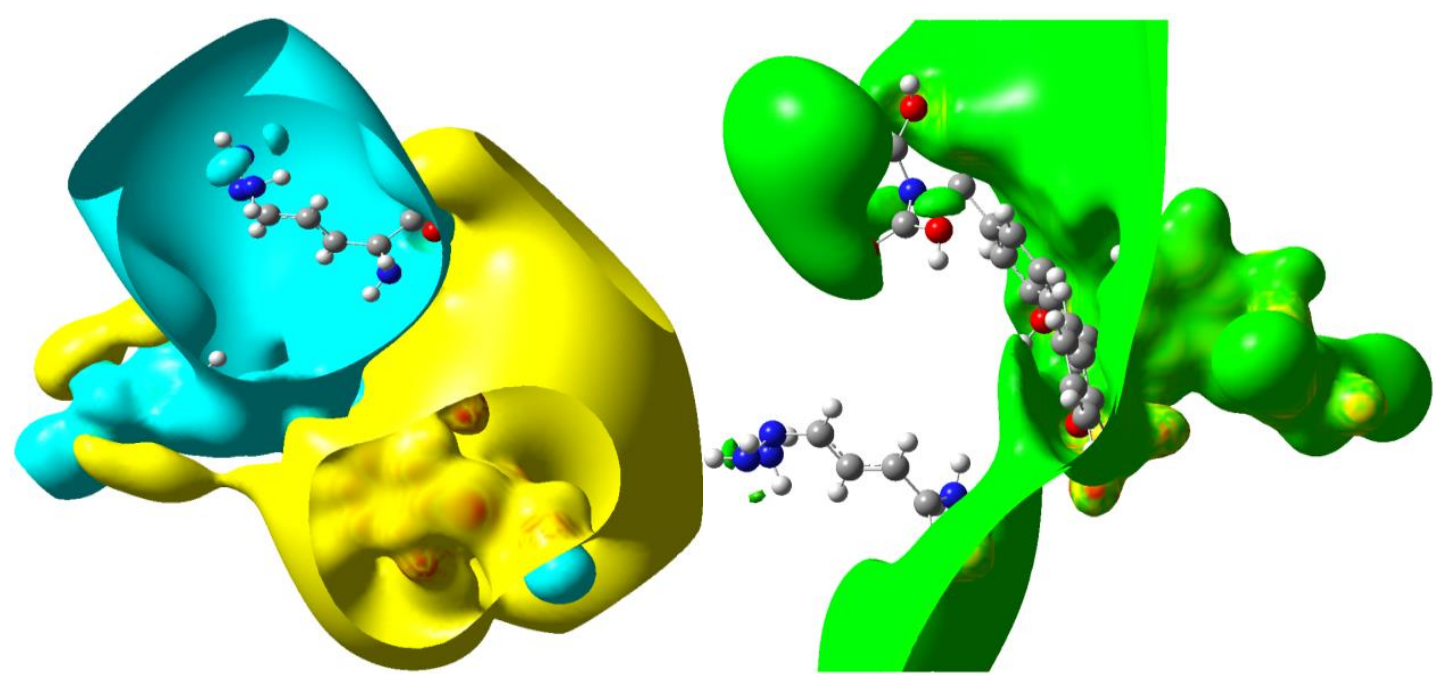

Figure 5. Different orientations of total electron density of LAP. 


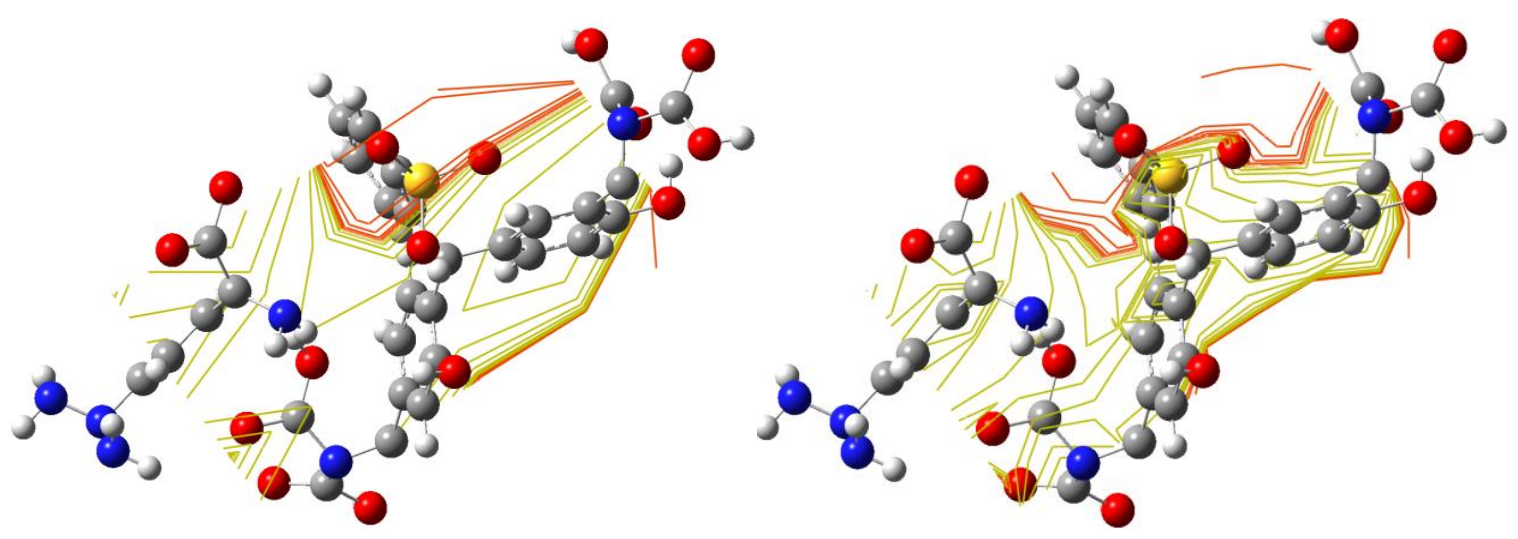

Figure 6. Different counter-mapping surfaces of LAP.

\section{Conclusions}

It is true based on the literature value mentioned above. Except for the carbon atoms, the title molecule LAP matches the above literature data in the current study. The presence of an electronegative atom pulls all carbon atom electron clouds to the $\mathrm{O}$ atom, resulting in carbon atom deshielding and a rise in chemical shift values. Based on the local block eigenvectors of the one-particle density matrix, NBO analysis was developed as a technique for examining hybridization and covalency effects in polyatomic wave functions. Localized bonds and lone pairs as basic components of molecular structure would be closely resembled by NBOs. These interactions are referred to as 'delocalization' corrections to the natural Lewis structure because they cause occupancy loss from the localized NBOs of the idealized Lewis structure into the empty non-Lewis orbitals (and therefore departures from the idealized Lewis structure description.

\section{Funding}

This research received no external funding.

\section{Acknowledgments}

The authors are grateful to acknowledge the Head of the Department of Physics, TAGGAC, Tindivanam, provided the necessary facility to complete this work.

\section{Conflicts of Interest}

The authors declare no conflict of interest.

\section{References}

1. Dong, X.; Min-Hua, J.; Zhong-Ke, T. A new phase matchable nonlinear optical crystal-L-arginine phosphate monohydrate (LAP). Acta Chim. Sin. 1983, 1, 230-233, https://doi.org/10.1002/cjoc.19830010217.

2. Notake, T.; Takeda, M.; Okada, S.; Hosobata, T.; Yamagata, Y.; Minamide, H. Characterization of all secondorder nonlinear-optical coefficients of organic N-benzyl-2-methyl-4-nitroaniline crystal. Sci. Rep. 2019, 9, 14853-8, https://doi.org/10.1038/s41598-019-50951-1.

3. Singh, A.K.; Sharma, R.; Singh, M.; Verma, N. Electrochemical determination of L-arginine in leukemic blood samples based on a polyaniline-multiwalled carbon nanotube-magnetite nanocomposite film modified glassy carbon electrode. Instrum. Sci. Technol. 2020, 48, 400416,https://doi.org/10.1080/10739149.2020.1734934. 
4. Latha, B.; Kumaresan, P.; Nithiyanantham, S.; Sampathkumar, K. Spectroscopic, Homo-Lumo and NLO studies of tetra fluoro phthalate doped Coumarin crystals using DFT method. J. Mol. Struct. 2017, 1142, 255260, http://dx.doi.org/10.1016/1.molstruc.2017.04.078.

5. Dolzhenkova, E.; Kostenyukova, E.; Bezkrovnaya, O.; Pritula, I. Effect of doping of KDP crystal with amino acid 1-arginine on the strength properties and character of laser damage. J. Cryst. Growth 2017, 478, 111116, https://doi.org/10.1016/j.jcrysgro.2017.08.010.

6. Kucherenko, I.; Soldatkin, O.; Dzyadevych, S.; Soldatkin, A. Electrochemical biosensors based on multienzyme systems: Main groups, advantages and limitations - A review. Anal. Chim. Acta 2020, 1111, 114-131, https://doi.org/10.1016/j.aca.2020.03.034.

7. Bollella, P.; Katz, E. Biosensors-Recent Advances and Future Challenges. Sensors 2020, 20, 6645, https://doi.org/10.3390/s20226645.

8. Naresh, V.; Lee, N. A Review on Biosensors and Recent Development of Nanostructured Materials-Enabled Biosensors. Sensors 2021, 21, 1109, https://doi.org/10.3390/s21041109.

9. Senthamizhan, A.; Sambathkumar, K.; Nithiyanantham, S. Physico-chemical, structural, optical and NLO studies on pure and La3+ doped 1-arginine acetate crystals. Mater. Sci. Energy Technol. 2020, 3, 282-288, https://doi.org/10.1016/j.mset.2019.11.005.

10. Nayl, A.; Abd-Elhamid, A.; El-Moghazy, A.Y.; Hussin, M.; Abu-Saied, M.; El-Shanshory, A.A.; Soliman, H.M. Thenanomaterials and recent progress in biosensing systems: A review. Trends Environ. Anal. Chem. 2020, 26, e00087, https://doi.org/10.1016/j.teac.2020.e00087.

11. Stasyuk, N.; Smutok, O.; Demkiv, O.; Prokopiv, T.; Gayda, G.; Nisnevitch, M.; Gonchar, M. Synthesis, Catalytic Properties and Application in Biosensorics of Nanozymes and Electronanocatalysts: A Review. Sensors 2020, 20, 4509, https://doi.org/10.3390/s20164509.

12. Gayda, G.; Demkiv, O.; Gurianov, Y.; Serkiz, R.; Klepach, H.; Gonchar, M.; Nisnevitch, M. "Green"Prussian Blue Analogues as Peroxidase Mimetics for Amperometric Sensing and Biosensing. Biosens. 2021, 11, 193, https://doi.org/10.3390/bios11060193.

13. Pritula, I.; Kostenyukova, E.; Bezkrovnaya, O.; Kolybaeva, M.; Sofronov, D.; Dolzhenkova, E.; Kanaev, A.; Tsurikov, V. KDP crystal doped with L-arginine amino acid: growth, structure perfection, optical and strength characteristics. Opt. Mater. 2016, 57, 217-224, https://doi.org/10.1016/j.optmat.2016.04.044.

14. Senthamizhan, A.; Sambathkumar, K.; Nithiyanantham, S.; Alagiriswamy, A.A. Electrical, Optical, Structural Properties with Some Physico-Mechanical of Pure and La3+ Doped L-Alanine Acetate Single Crystals. Sens. Lett. 2020, 18, 894-899, https://doi.org/10.1166/s1.2015.3500.

15. Fonseca, T.L.; Sabino, J.R.; Castro, M.; Georg, H.C. A theoretical investigation of electric properties of Larginine phosphate monohydrate including environment polarization effects. J. Chem. Phys. 2010, 133, 144103, https://doi.org/10.1063/1.3501237.

16. Kostenyukova, EI.; Pritula, IM.; Bezkrovnaya, ON.; Kovalenko, NO.; Doroshenko1, AG.; Khimchenko, SV.; Fedorov, AG. Effect of L-arginine phosphate doping on structural, optical and strength properties of KDP single crystal, SPQEO, 2019, 22, 60-66, https://doi.org/10.15407/spqeo22.01.60.

17. Dave, D.J.; Parikh, K.D.; Joshi, M.J. Vickers Micro-Hardness Studies of Amino Acids (L-Histidine, LThreonien and DL-Methionine) Doped KDP Crystals. Adv. Mater. Res. 2013, 665, 172-178, https://doi.org/10.4028/www.scientific.net/AMR.665.172.

18. Kostenyukova, E. Microhardness, laser damage threshold and SHG efficiency studies of potassium dihydrogen phosphate crystals doped with L-arginine amino acid. Funct. Mater. 2016, 23, 27-31, http://dspace.nbuv.gov.ua/handle/123456789/119715.

19. Thirupugalmani, K.; Venkatesh, M.; Karthick, S.; Maurya, K.K.; Vijayan, N.; Chaudhary, A.K.; Brahadeeswaran, S. Influence of polar solvents on growth of potentially NLO active organic single crystals of N-benzyl-2-methyl-4-nitroaniline and their efficiency in terahertz generation. CrystEngComm 2017, 19, 2623-2631, https://doi.org/10.1039/C7CE00228A.

20. Demkiv, O.; Stasyuk, N.; Serkiz, R.; Gayda, G.; Nisnevitch, M.; Gonchar, M. Peroxidase-Like Metal-Based Nanozymes: Synthesis, Catalytic Properties, and Analytical Application. Appl. Sci. 2021, 11, 777, https://doi.org/10.3390/app11020777.

21. Muley, G.; Rode, M.; Pawar, B. FT-IR, Thermal and NLO Studies on Amino Acid (L-Arginine and LAlanine) Doped KDP Crystals. Acta Phys. Pol. A 2009, 116, 1033-1038, https://doi.org/10.12693/aphyspola.116.1033.

22. Гайда, Г. ОКСИДАЗИ L-АМІНОКИСЛОТ: ОДЕРЖАННЯ, ВЛАСТИВОСТІ ТА ПЕРСПЕКТИВИ ВИКОРИСТАННЯ. InterConf 2021, 63, 228-237, https://doi.org/10.51582/interconf.21-22.06.2021.25. 
23. Arjun, K.; Ganesh, D.; Karthick, S.; Santha, A.; Venkatesh, M.; Ganesamoorthy, S.; Brahadeeswaran, S.; Chaudhary, A. Investigations on aqueous solution grown 1-Arginine Phosphate monohydrate (LAP) single crystals for efficient $\mathrm{THz}$ generation. J. Cryst. Growth 2021, 575, 126337, https://doi.org/10.1016/j.jcrysgro.2021.126337.

24. Kooliyankal, N.; Sreedharan, R.; Ravi, S.; Ravindran, R.K.; Krishnankutty, M.K.T. Experimental and computational studies on optical properties of a promising N-benzylideneaniline derivative for nonlinear optical applications. Zeitschriftfür Naturforschung A 2020, 75, 557-573, https://doi.org/10.1515/zna-20200047.

25. Mahadik, A.; Soni, P.H.; Chaudhari, K. Habit modification in L-arginine doped potassium dihydrogen phosphate crystal growth under applied electric field. In Proceedings of the DAE SOLID STATE PHYSICS SYMPOSIUM 2019; AIP Publishing, 2020; p. 2265, https://doi.org/10.1063/5.0016880.

26. Senthilkuamr, C.; Srinivasan, M. Optical Properties of Single Crystals, 2021, 103-290. http://dx.doi.org/10.5772/intechopen.75181. 